\title{
Concepciones de aprendizaje desde una perspectiva fenomenográfica. Convergencias y diferencias entre estudiantado universitario portugués y latinoamericano ${ }^{1}$
}

\section{Learning Conceptions From a Phenomenographic Approach. Convergences and Differences Between Portuguese and Latin American University Students ${ }^{2}$}

\section{Concepções de aprendizado desde uma perspectiva fenomenográfica. Convergências e diferenças entre estudantes universitários portugueses e latino-americanos ${ }^{3}$}

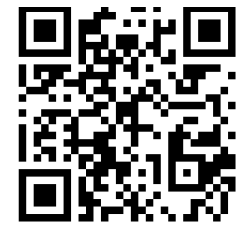

Ida Lucía Morchio Universidad Nacional de Cuyo Facultad de Filosofía y Letras Mendoza, Argentina lucy_morchio@yahoo.com.ar (D) https://orcid.org/0000-0002-1072-5019

Solange Astudillo Universidad Nacional de Cuyo Facultad de Filosofía y Letras Mendoza, Argentina sol.astu@gmail.com https://orcid.org/0000-0002-7540-9009

Hilda Difabio Consejo Nacional de Investigaciones Científicas y Técnicas Mendoza, Argentina ganglat@gmail.com

Recibido • Received • Recebido: 24 / 10 / 2018

Corregido • Revised • Revisado: 11 / 12 / 2019

Aceptado • Accepted • Aprovado: 01 / 04 / 2020

\footnotetext{
${ }^{1}$ Código de proyecto de investigación 06/G746. SeCTyP, UNCuyo.

2 Research Project code 06/G746. SeCTyP, UNCuyo.

${ }^{3}$ Código do Projeto de pesquisa 06/G746. SeCTyP, UNCuyo.
} 
http://doi.org/10.15359/ree.24-2.10

ROR: https://ror.org/01t466c14 Universidad Nacional, Costa Rica

http://www.una.ac.cr/educare

educare@una.cr

Resumen: Este artículo reseña un estudio en el que se indagan las concepciones de aprendizaje en estudiantado latinoamericano que cursa una misma carrera (Ciencias de la Educación/Pedagogía), a fin de poner en relación los resultados con los obtenidos por Rosário et ál. (2006) en Portugal. El objetivo de la investigación que se informa es ponderar los alcances y límites de aplicar el sistema categorial de las personas autoras nombradas a las concepciones de aprender extraídas en diferentes contextos. Ambas investigaciones comparten un enfoque fenomenográfico, pero difieren en la población y en el instrumento. La nuestra se realizó en 264 estudiantes que cursaban el tramo inicial o final de la carrera en Argentina (Universidad Nacional de Cuyo), Brasil (Universidad Federal de Río de Janeiro y Universidad Estadual Paulista) y México (Universidad de Colima), mediante un ítem abierto del Inventario de concepciones y experiencias de aprender en la universidad (INCEAPU). El trabajo de Rosário et ál. (2006) se realizó en 16 estudiantes de la Universidad de Évora a través de entrevistas individuales. Los hallazgos ponen de manifiesto algunas concepciones compartidas, otras semejantes, pero no idénticas y otras distintivas de la muestra en análisis, lo que lleva a concluir que, si bien se reconocen similitudes y solapamientos, también se destacan particularidades en función del contexto educativo. Por otra parte, sugieren que las concepciones adquieren un sentido bidireccional, pues aunque se inician en el estudiantadoy condicionan su actuar frente a la propuesta didáctica, dicha propuesta incide en lo que el estudiantado entiende por aprender. Es relevante indagar las concepciones de aprendizaje en las palabras del alumnado porque, si la universidad se compromete con el respeto por la diversidad, lo primero es conocer en qué consiste "lo diverso" en términos de las diferentes formas en que sus estudiantes entienden lo que es aprender.

Palabras claves: Concepciones de aprendizaje; universidades; estudiantado universitario portugués y latinoamericano; implicaciones didácticas.

Abstract: This paper describes a study about the empirical conceptions that a group of Latin American students, attending the same career (Learning sciences/Pedagogy), has about learning to relate the results with those obtained by Rosário et al. (2006) in Portugal. This research aims to weigh the scopes and limits of applying the categorial system developed by the authors to conceptions of learning obtained from different contexts. Both research works share a phenomenological approach, but they differ in population and instrument. Our research was conducted on 264 students -attending the initial or the final courses of their careers in Argentina (National University of Cuyo), Brazil (Federal University of Rio de Janeiro and Paulista State University) and Mexico (University of Colima)-, using an open item of the Inventory of Learning Conceptions and Experiences at University (INCEAPU). The work of Rosário et al. (2006) was applied to 16 students of the University of Évora through individual interviews. Findings show shared conceptions, some similar but not identical categories, and other categories that are different from the sample under analysis. Therefore, it can be concluded that, although some similarities and overlaps can be observed, there are also distinctive aspects, according to the educational context. On the other hand, findings suggest that, even though conceptions start on the students' community and condition their response to the educational tasks, such conceptions become bidirectional, since the teaching methods influence students' perception of learning. It is relevant to research the learning conceptions in students' words because, if the university makes a commitment involving respect for diversity, the first issue in question is to know what is "the diverse" in terms of the different ways in which students understand the concept of learning.

Keywords: learning conceptions; universities; Portuguese and Latin American university students; educational implications. 
Resumo: Este artigo resenha um estudo empírico no qual são indagadas as concepções de aprendizado em estudantes latino-americanos que cursam uma mesma carreira (Ciências da educação/ Pedagogia), a fim de pôr em relação os resultados com os obtidos por Rosário et al. (2006) em Portugal. O objetivo da investigação é ponderar os alcances e limites de aplicar o sistema categorial dos autores nomeados às concepções de aprender, extraídas em diferentes contextos. Ambas investigações compartem um enfoque fenômenográfico, mas diferem em quanto população e instrumento. A nossa foi realizada em 264 estudantes que cursavam o trajeto inicial ou final da carreira na Argentina (Universidade Nacional de Cuyo), no Brasil (Universidade Federal do Río de Janeiro e Universidade Estadual Paulista) e no México (Universidade de Colima), por meio de um item aberto do Inventário de Concepções e Experiências de Aprender na Universidade (INCEAPU). O trabalho de Rosário et al. (2006) foi realizado com 16 estudantes da Universidade de Évora através de entrevistas individuais. As descobertas põem de manifesto algumas concepções compartilhadas, outras semelhantes, mas não idênticas e outras diferentes da amostra em análise, o que leva a concluir que, se bem se reconhecem semelhanças e sobreposições, também se destacam particularidades em função do contexto educativo. Por outra parte, sugerem que as concepções adquiram um sentido bidirecional porque, a pesar de que se iniciam na pessoa estudante e condicionam seu proceder perante a proposta didática, tal proposta incide no que se entende por aprender. É relevante indagar as concepções de aprendizagem nas palavras dos estudantes porque, se a universidade se compromete com o respeito à diversidade, o primeiro a fazer é conhecer em quê consiste "a diversidade" em termos das diferentes formas em que os e as estudantes entendem o que é aaprender.

Palavras chaves: Concepções de aprendizado; universidades; estudantes universitários portugueses e latino-americanos, implicações didáticas.

\section{Introducción}

Las concepciones de aprendizaje remiten a los modos en que el estudiantado entiende, experimenta y da sentido al aprendizaje (Cano, 2005). A la base se encuentran esquemas de conocimiento generales y conocimientos ligados a entornos y circunstancias particulares, en nuestro caso, académicos (Morchio y Del Río, 2015). Estas concepciones no constituyen ideas aisladas, sino que subyacen a las conductas y actitudes del individuo (Säljö, 1979).

Indagar sobre lo que entiende el estudiante cuando dice aprender es relevante por su incidencia en el modo en que el aprendiz organiza su obrar y anticipa lo que deberá lograr para mostrarle al profesorado como prueba de que efectivamente aprendió un contenido de estudio.

En este artículo ponemos en relación, por un lado, resultados de investigaciones sobre concepciones de aprendizaje en una muestra integrada por estudiantado argentino, brasileño y mexicano que cursa Ciencias de la Educación o Pedagogía consideradas carreras equivalentes (en función del objeto de estudio, el contenido de aprendizaje y el perfil de egreso) realizadas en una línea de trabajo sobre Aprendizaje en la universidad desarrollada en la Universidad Nacional de Cuyo (UNCuyo) (Mendoza, Argentina) (Morchio, 2016-2018); y, por otro lado, los resultados informados por Rosário et ál. (2006), en el artículo denominado Perspectiva fenomenográfica de las concepciones del aprendizaje, cuyos objetivos son: 1) explorar las diversas concepciones de aprendizaje del estudiantado y su estructura; y 2) identificar las semejanzas y diferencias entre las concepciones de alumnado de diferentes cursos. 
http://doi.org/10.15359/ree.24-2.10

ROR: https://ror.org/01t466c14 Universidad Nacional, Costa Rica

http://www.una.ac.cr/educare

educare@una.cr

Seleccionamos este estudio realizado en la Universidad de Évora porque comparte con el nuestro la aproximación a las concepciones de aprendizaje desde la subjetividad de sus actores; no obstante, los dos estudios difieren respecto de sus participantes y del instrumento.

Rosário et ál. (2006) trabajaron en una muestra conformada por estudiantes de centros educativos públicos de Portugal, en la que estaba representado alumnado de enseñanza básica, de secundaria y de nivel universitario. A fines de nuestro trabajo comparativo, la investigación que informamos tuvo en cuenta 16 estudiantes del nivel universitario (Licenciatura en Formación de Profesorado de la Universidad de Évora).

En entrevistas individuales, preguntaron: ¿Qué es aprender? El análisis mostró el "número de formas cualitativamente distintas de percibir el fenómeno de aprendizaje" (Rosário et ál., 2006, p. 198), y posibilitó construir categorías que describen las concepciones de aprendizaje en la muestra de estudiantes portugueses.

En la investigación realizada en la UNCuyo, entre 2007y 2018, las concepciones de aprendizaje se recopilaron mediante un ítem abierto del Inventario de concepciones y experiencias de aprender en la universidad (INCEAPU) (Morchio y Difabio de Anglat, 2018). El corpus lo conforman 501 producciones escritas por estudiantes del tramo inicial y final de cinco carreras -Ciencia Política y Administración Pública, Medicina, Ciencias de la Educación, Trabajo Social y Pedagogíaen cuatro universidades: UNCuyo, de Argentina, la UFRJ y la UEP, de Brasil, y la UdC de México4.

El objetivo general de la investigación que se informa es ponderar los alcances y límites de aplicar el sistema categorial de Rosário et ál. (2006) a las concepciones de aprender extraídas en una muestra de estudiantado latinoamericano. Por ende, nuestros objetivos específicos son: 1) describir el comportamiento del sistema de categorías de Rosário et ál. (2006); y 2) identificar convergencias y diferencias en las concepciones de aprendizaje entre estudiantado latinoamericano y portugués.

Las categorías de Rosário et ál. (2006) son catorce. Aprender es: 1) algo envolvente y diversificado, 2) aumentar el conocimiento, 3) memorizar, 4) aplicar, 5) comprender, 6) ver las cosas de forma diferente, 7) cambiar como persona, 8) realizarse, 9) un proceso no limitado por el tiempo o el espacio, 10) un proceso individualizado, 11) un proceso experiencial, 12) un proceso interactivo, 13) un proceso de enseñanza y 14) positivo.

El presente artículo se organiza en seis secciones. Se parte del estado de la cuestión, en el que se reseñan trabajos relevantes sobre el tema. En segundo lugar, se explicitan las bases teóricas de la investigación que se comunica. Luego, se describe su metodología (participantes, procedimiento, instrumento y estrategia para el procesamiento de los datos). El apartado siguiente, análisis y discusión de los resultados, incluye: a) comportamiento de cada categoría de

\footnotetext{
${ }^{4}$ UFRJ: Universidad Federal de Río de Janeiro, UEP: Universidad Estadual Paulista, UdC: Universidad de Colima.
} 
concepciones de aprendizaje de Rósario et ál. (2006) respecto de las concepciones de aprendizaje identificadas en la población latinoamericana; b) nuevas formas de entender el aprendizaje, solapamientos y situaciones particulares emergentes de la comparación; y c) convergencias y divergencias en las concepciones de aprendizajeentre estudiantadolatinoamericanoy portugués. Las conclusiones recuperan las discrepancias halladas y muestran el riesgo de considerar, sin más, que las concepciones son transversales, generalizables a diferentes contextos académicos. Finalmente, se derivan algunas implicaciones para el plano aplicado.

\section{Estado de la cuestión}

En el campo de los estudios cualitativos en psicología de la educación, Marton y Säljö (1976) indagan las estrategias de aprendizaje con las se compromete el alumnado universitario (de Suecia) en una tarea de comprensión de texto. Hallan dos niveles de procesamiento que se distinguen claramente en función de la tarea de aprender el texto: superficial vs. profundo. En el primero, el alumnado dirige su atención hacia los signos y se atiene a una estrategia de aprendizaje memorístico. En el caso del procesamiento de nivel profundo, el sujeto lector se ocupa del significado, "se dirige a la comprensión de lo que el autor quiere decir" (p. 8). Este trabajo inicia una de las líneas de estudio de los denominados estilos de aprendizaje, temática que excede los límites del nuestro.

Por su parte, las concepciones de aprendizaje cuentan con un amplio cuerpo de investigaciones; entre ellas, desde un enfoque fenomenográfico, es seminal el trabajo con estudiantado universitario realizado por Marton et ál. (1993) y Säljö (1979), quienes identificaron seis categorías que se constituyeron en punto de arranque para estudios posteriores: 1) aumento de conocimiento, 2) memorización y reproducción, 3) adquisición de hechos y métodos que pueden ser aplicados, 4) comprensión de significados, 5) interpretación de la realidad y 6) transformación de la persona. En las tres primeras, prevalece un cambio cuantitativo (adición de información), mientras que en las restantes el aprendizaje supone aspectos cualitativos (comprensión del sentido y transformación en el contenido o en quien aprende) (Cano, 2005).

Desde el enfoque de las teorías implícitas, Pozo y Scheuer (1999) reconocen tres concepciones sobre el aprendizaje -directa, interpretativa y constructiva-, las que se distinguen entre sí respecto de lo que se aprende (contenidos), cómo se aprende (procesos implicados en la adquisición) y condiciones (variables externas que influyen en él) (Morchio y Del Río, 2015).

Martínez-Fernández (2007), en 276 estudiantes que se encuentran en años iniciales, medios y finales de la carrera de Psicología en la Universidad de Barcelona (España), aplica y corrobora las tres categorías de Pozo y Scheuer (1999).

Rosário et ál. (2006), en la muestra de 16 estudiantes de universidades portuguesas, identifican catorce categorías, algunas coincidentes con las mencionadas en Marton et ál. (1993) y Säljö (1979) y otras propias. Rosário et ál. (2006) amplían dicho sistema de categorías 
y organizan las concepciones de aprender que hallaron en estudiantes portugueses, según cuatro enfoques: 1. externo (incrementar el conocimiento, memorizar y aplicar), 2. interno y transformativo (comprender, ver las cosas de forma diferente, cambiar como persona, realizarse), 3. de proceso (proceso no limitado en espacio y tiempo, proceso individualizado, proceso experiencial, proceso interactivo y proceso formativo) y 4. de valor (aprender es positivo).

Como corolario, señalan que el aprendizaje es conceptualizado en forma mayoritaria como un incremento de conocimientos.

En la UNCuyo, desde 2007 se estudia el Aprender en funcionamiento, en el marco de cinco proyectos de investigación consecutivos. Se indaga sobre concepciones, experiencias y autopercepción de competencia para aprender, tal como son vivenciadas e informadas por sus protagonistas. En uno de los proyectos se recopilaron las concepciones de aprendizaje en estudiantes de cinco carreras ( $N=501$ ) y en el análisis efectuado en 2015 se observaron diferencias claras según carrera y menos claras según tramo (Morchio y Del Río, 2015). Los resultados obtenidos constituyen antecedentes de la investigación que se informa en el presente artículo.

\section{Bases teóricas}

La línea de investigación en desarrollo en la UNCuyo se inscribe en el campo de la psicología educacional y se sustenta en postulados cognitivistas/constructivistas, complementados con la experiencia de las autoras en docencia y orientación con estudiantado universitario.

Con una mirada cognitivista, nos preguntamos quépiensa el estudiantado universitario cuando dice aprender, haciéndonos eco del comentario de Bruner (2001, p. 264), quien advierte que a veces "usamos las mismas palabras pero no poseemos los mismos conceptos". Ello anticipa posibles fricciones entre lo que entiende por aprender cada protagonista principal del encuentro educativo.

Por otra parte, en la dinámica de la autorregulación de la conducta, las concepciones de aprendizaje operan como un marco de referencia epistémico (Marton y Säljö, 1976) con la credibilidad suficiente para guiar las acciones. En consecuencia, la expectativa es contribuir a la autonomía con el aporte de insumos que permitan comprender el aprender como proceso activo y sistema complejo (Morchio y Del Río, 2015).

En particular, referido al tema concepciones de aprendizaje, tenemos en cuenta los estudios desarrollos por Cuberos Pérez et ál. (2019), Martínez-Fernández (2007), Marton et ál. (1993), Pozo y Scheuer (1999), Pozo et ál. (2009) y Säljö (1979).

\section{Metodología}

El estudio sobre concepciones de aprendizaje que se informa en este artículo implementa una aproximación fenomenográfica. Forma parte de la línea de investigación sobre Aprendizaje 
en la Universidad desarrollada en forma ininterrumpida entre 2007 y 2018 en la UNCuyo (Morchio, 2007-2018). La línea incluye cinco proyectos bienales avalados y financiados por la SeCTyP (Secretaría de Ciencia, Técnica y Posgrado) de la UNCuyo, en los que participó estudiantado argentino, brasileño y mexicano que cursa el $2^{\circ}$ y el último año (tramo inicial/final) de Ciencias de la Educación o Pedagogía en la UNCuyo, la UFRJ, la UEP ${ }^{5}$ y la UdC. Las autoras de este artículo integran el equipo de investigación.

Participaron 264 estudiantes, 138 del tramo inicial y 126 del tramo final, según se detalla en la Tabla 1.

Tabla 1: Distribución de los participantes según país, institución, carrera y tramo

\begin{tabular}{|c|c|c|c|c|c|}
\hline País & Universidad & Carrera & Año de toma & Tramo & $N=$ \\
\hline \multirow{2}{*}{ Argentina } & \multirow{2}{*}{ Universidad Nacional de Cuyo } & \multirow{2}{*}{ Ciencias de la Educación } & 2011 & Inicial & 29 \\
\hline & & & 2014-2015 & Final & 29 \\
\hline \multirow{2}{*}{ México } & \multirow{2}{*}{ Universidad de Colima } & \multirow{2}{*}{ Pedagogía } & \multirow{2}{*}{2013} & Inicial & 55 \\
\hline & & & & Final & 40 \\
\hline \multirow{2}{*}{ Brasil } & \multirow{2}{*}{ Universidad Federal de Río de Janeiro } & \multirow{2}{*}{ Pedagogía } & 2010 & Inicial & 30 \\
\hline & & & 2014 & Final & 45 \\
\hline & \multirow{2}{*}{ Universidad Estadual Paulista } & \multirow{2}{*}{ Pedagogía } & \multirow{2}{*}{2014} & Inicial & 24 \\
\hline & & & & Final & 12 \\
\hline
\end{tabular}

Nota: Elaboración propia.

Los datos se reunieron entre 2010 y 2015, mediante la administración del INCEAPU en situación regular de clase, por integrantes del equipo de investigación. El ítem 3 de este cuestionario de autoinforme solicita una producción escrita en respuesta a la consigna: Explica en un párrafo qué es aprender para vos. Desde el enunciado se anticipa que se toma en cuenta cómo el estudiantado entiende el aprender, aunque no necesariamente coincida con su forma de ponerlo en obra.

El procesamiento se formalizó en cuatro etapas: Primera, en la investigación realizada en la UNCuyo, las producciones escritas del estudiantado (el corpus) se analizaron por universidad y, dentro de cada una de ellas, según tramo (inicial/final); por esa vía se extrajeron las categorías en vivo. Segunda, se recuperaron las concepciones identificadas por Rosário et ál. (2006) en estudiantes de la Universidad de Évora. Tercera, se pusieron en relación los resultados de ambos estudios y se observaron las convergencias y divergencias en lo que entienden por aprender estudiantes de la muestra latinoamericana y de la muestra portuguesa. Cuarta, se consideraron categorías más globales que emplean Rosário et ál. (2006, pp. 22-23) para analizar el aprender según tres focos: extensión y diversidad, instrumental y transformativo.

\footnotetext{
${ }^{5}$ Las producciones originales en portugués fueron traducidas al español por una traductora pública de la UNCuyo.
} 
En estas etapas, a fin de respaldar la consistencia de los resultados, las dos primeras autoras del presente artículo trabajaron colaborativamente en una suerte de validación interjuezas, deliberando sobre los casos en que diferían hasta alcanzar un acuerdo firme.

Durante el análisis, se presentaron dos limitaciones. Por una parte, la investigación realizada en Portugal, como ya señalamos, incluyó estudiantes de tres niveles educativos y, en la comunicación de sus resultados, varias concepciones se ejemplifican con producciones recogidas en estudiantes de $9^{\circ}$ y $12^{\circ}$ años; mientras, en nuestro caso, solo consideramos el grupo universitario. Por otra parte, no refiere a la frecuencia con que se muestra cada concepción. A pesar de estas limitaciones, nos interesó este sistema de categorías por la amplitud de perspectivas que incluye.

\section{Análisis y discusión de los resultados}

La presentación de los resultados y su análisis se organiza en función de las concepciones de aprendizaje halladas por Rosário et ál. (2006). Los resultados para cada una de las catorce categorías de estas personas autoras se describen, primero, según el estudio realizado en Portugal y, segundo, según los obtenidos en la muestra latinoamericana, ilustrados por medio de palabras textuales del estudiantado ${ }^{6}$.

\section{Relación entre las concepciones de aprendizaje en estudiantado portugués y latinoamericano}

En función de una lógica comparativa, analizamos cómo se muestra cada una de las categorías de Rosário et ál. (2006) en la muestra latinoamericana, según universidad y tramo.

Las características de esta investigación-por un lado, 264 estudiantes, cuatro universidades, dos tramos y, por otro, un sistema de catorce categorías in vitro- repercuten en la complejidad del análisis. Por tal motivo, y sin perder de vista el enfoque cualitativo (fenomenográfico) del estudio, se incluyen también, instancias de cuantificación como recurso para precisar las apreciaciones en términos de mayor o menor presencia por contexto educativo y por tramo. Esta estrategia posibilita una visión panorámica de la situación, la que admite numerosas lecturas, y la oportunidad de poner en diálogo nuestros resultados con los que informan otros estudios sobre la temática.

\footnotetext{
${ }^{6}$ La referencia que identifica cada producción se compone de tres elementos: universidad, tramo y caso. Por ejemplo, en "UFRJ-TF-C35", UFRJ significa Universidad Federal de Río de Janeiro; TF significa tramo final (alumnos que cursan el último año de la carrera) y C35, número de caso dentro de la universidad y el tramo.
} 
En la Tabla 2 se muestra la frecuencia en que cada categoría de Rosário et ál. (2006) se halló en las producciones del estudiantado que participa en nuestro estudio.

Tabla 2: Frecuencia de las categorías de Rosário et al. (2006) en la muestra latinoamericana por carrera y por tramo

\begin{tabular}{|c|c|c|c|c|c|c|c|c|c|c|c|c|}
\hline & \multicolumn{2}{|c|}{ UNCuyo } & \multicolumn{2}{|c|}{ UEP } & \multicolumn{2}{|c|}{ UFRJ } & \multicolumn{2}{|c|}{ UdC } & \multicolumn{2}{|c|}{ Total por tramo } & \multirow{3}{*}{$\begin{array}{c}\begin{array}{c}\text { Total } \\
\text { categoría }\end{array} \\
\mathbf{2 6}\end{array}$} \\
\hline & & \multirow{2}{*}{$\begin{array}{c}\text { Tramo } \\
\text { inicial } \\
4 \\
\end{array}$} & \multirow{2}{*}{$\begin{array}{c}\begin{array}{c}\text { Tramo } \\
\text { final }\end{array} \\
10\end{array}$} & \multirow{2}{*}{$\begin{array}{c}\text { Tramo } \\
\text { inicial }\end{array}$} & \multirow{2}{*}{$\begin{array}{c}\begin{array}{c}\text { Tramo } \\
\text { final }\end{array} \\
0\end{array}$} & \multirow{2}{*}{$\begin{array}{c}\text { Tramo } \\
\text { inicial }\end{array}$} & \multirow{2}{*}{$\begin{array}{c}\text { Tramo } \\
\text { final }\end{array}$} & \multirow{2}{*}{$\begin{array}{c}\begin{array}{c}\text { Tramo } \\
\text { inicial }\end{array} \\
3\end{array}$} & \multirow{2}{*}{$\begin{array}{c}\text { Tramo } \\
\text { final } \\
0\end{array}$} & \multirow{2}{*}{$\begin{array}{c}\text { Tramo } \\
\text { inicial }\end{array}$} & \multirow{2}{*}{$\begin{array}{c}\begin{array}{c}\text { Tramo } \\
\text { final }\end{array} \\
\mathbf{1 3}\end{array}$} & \\
\hline 1 & Envolvente & & & & & & & & & & & \\
\hline 2 & Adquirir & 3 & 9 & 7 & 3 & 12 & 13 & 34 & 33 & 56 & 58 & 114 \\
\hline 3 & Memorizar & 1 & 1 & 1 & 0 & 0 & 1 & 12 & 3 & 14 & 5 & 19 \\
\hline 4 & Aplicar & 6 & 4 & 2 & 2 & 4 & 1 & 16 & 13 & 28 & 20 & 48 \\
\hline 5 & Comprehender & 19 & 6 & 17 & 4 & 7 & 16 & 16 & 10 & 59 & 36 & 95 \\
\hline 6 & Ver diferente & 2 & 8 & 0 & 2 & 4 & 9 & 1 & 2 & 7 & 21 & 28 \\
\hline 7 & $\begin{array}{l}\text { Cambiar como } \\
\text { persona }\end{array}$ & 9 & 5 & 4 & 0 & 2 & 4 & 0 & 3 & 15 & 12 & 27 \\
\hline 8 & Realizarse-futuro & 2 & 0 & 2 & 1 & 0 & 0 & 0 & 0 & 4 & 1 & 5 \\
\hline 9 & $\begin{array}{l}\text { No limitado por } \\
\text { espacio y tiempo }\end{array}$ & 10 & 7 & 1 & 5 & 1 & 1 & 4 & 4 & 16 & 17 & 33 \\
\hline 10 & Individualizado & 8 & 8 & 2 & 0 & 2 & 0 & 2 & 0 & 14 & 8 & 22 \\
\hline 11 & Experiencial & 0 & 0 & 1 & 0 & 2 & 4 & 1 & 1 & 4 & 5 & 9 \\
\hline 12 & Interactivo & 0 & 3 & 3 & 3 & 0 & 6 & 3 & 0 & 6 & 12 & 18 \\
\hline 13 & Enseñanza & 0 & 2 & 1 & 2 & 2 & 4 & 4 & 7 & 7 & 15 & 22 \\
\hline \multirow[t]{3}{*}{14} & Valor positivo & 0 & 0 & 1 & 0 & 0 & 0 & 0 & 1 & 1 & 1 & 2 \\
\hline & Total por tramo & 64 & 63 & 42 & 22 & 42 & 62 & 96 & 77 & 244 & 224 & 468 \\
\hline & $\begin{array}{l}\text { Total por } \\
\text { universidad }\end{array}$ & \multicolumn{2}{|c|}{127} & \multicolumn{2}{|c|}{64} & \multicolumn{2}{|c|}{104} & \multicolumn{2}{|c|}{173} & \multicolumn{2}{|c|}{468} & \\
\hline
\end{tabular}

Nota: Elaboración propia.

${ }^{7}$ Hemos empleado el siguiente código cromático para favorecer la lectura: rojo = presencia destacada; verde = presencia notable; lila $=$ presencia moderada y celeste $=$ presencia mínima.

Un aspecto por tener presente es la composición de las muestras (cfr. Tabla 1): UNCuyo (29 estudiantes en cada tramo); UdC (inicial $N=55$, final $N=40$ ), UFRJ (inicial $N=30$, final $N=45$ ) y UEP (inicial $N=24$, final $N=12$ ). 
A continuación se describe cómo se manifiesta, en la muestra de estudiantado latinoamericano, cada una de las concepciones de aprendizaje identificadas en estudiantado portugués (Rosário et ál, 2006).

1. "Aprender es algo envolvente y diversificado, en cuanto a lo que se aprende, a la forma en que se aprende y a las fuentes de aprendizaje".

En el corpus de estudiantes de Latinoamérica lo que se aprende incluye conocimientos, hábitos, valores, saberes, teorías, experiencias, destrezas y costumbres: Aprender es un proceso que se da durante toda la vida, y consiste en la adquisición de hábitos, de conocimientos, de valores, etc., que nos forman como persona y que nos humaniza para desenvolvernos de modo correcto (UNCuyo-TI-C23). Con respecto a las fuentes, Se puede aprender a aprender en todo el entorno social, en el hogar y en tu centro de estudio (UdC-TI-45). Esta categoría de Rosário et ál. (2006) aparece en la muestra latinoamericana con un porcentaje bajo (5\% del total), en las respuestas del estudiantado argentino -principalmente en el tramo final-, en la UFRJ y la UdC -tramo inicial- y no se menciona en la UEP.

2. "Aprender es aumentar el conocimiento". La principal característica de esta concepción es la adquisición y posesión de conocimientos según una lógica informativa.

Una parte de la muestra latinoamericana también entiende el aprendizaje como asimilar, adquirir nuevos conocimientos. Aprender es adquirir un nuevo conocimiento que le es significativo a través de la clase, textos, práctica o por medios propios (UFRJ-TI-C5). Esta categoría es la que más se reitera en las producciones del estudiantado de las cuatro universidades y en ambos tramos ( $24 \%$ del total), con diferencias en el énfasis. Su presencia es destacada en ambos tramos en la UdC; lo es en menor proporción en la UFRJ en ambos tramos, en la UNCuyo en el tramo final y en la UEP en el tramo inicial.

En una mirada de conjunto (cfr. Tabla 2), se observa la diferencia entre universidades; no obstante, en el recuento total por tramo la proporción es semejante.

3. "Aprender es memorizar". En estudiantado portugués la memorización se plantea como una forma de almacenar la información, aunque no se la asocia, de modo explícito o implícito, con la reproducción o la comprensión.

En algunas producciones de nuestro estudio se hace referencia a incorporar en la memoria lo aprendido, en términos de memoria mecánica, mientras que otras vinculan memoria con comprensión. Aprender es que el conocimiento que adquieres lo tengas en tu memoria, pero de manera analizada, que sepas que es realmente lo que es, y que no sea un aprendizaje con cierta extensión de tiempo (UdC-TI-C12). Si bien esta categoría se muestra de modo explícito en pocas producciones (4\%), como en el caso anterior difiere entre contextos académicos: se reitera en la UdC, tramo inicial, mientras que, en los otros contextos y tramos, o no aparece o su presencia es mínima. 
4. "Aprender es aplicar". En la muestra de estudiantes de Portugal, una parte entiende el aprendizaje como uso de conocimientos o procedimientos, no restringido al contexto escolar sino que se extiende a diversas circunstancias y contextos en los que el sujeto se desempeña en el presente o lo hará en el futuro.

En la muestra latinoamericana esta categoría se expresa como capacidad de utilizar lo aprendido en diferentes situaciones, transferirlo. Es el proceso en el cual se adquieren saberes y se logra retener información que posteriormente se podrá aplicar en la vida escolar o en un entorno real (UdC-TF-C62). Se observa en un $10 \%$ del total en los cuatro contextos académicos, con mayor frecuencia en las producciones del estudiantado mexicano, seguidos -con sentido decreciente- por argentino y los brasileño.

Es interesante notar la diferencia de énfasis según contexto académico y la heterogeneidad entre tramos. En la UdC su presencia es notable en ambos tramos; en la UNCuyo-ambos tramosy la UFRJ -tramo inicial- es moderada; en la UEP es mínima en ambos tramos.

5. "Aprender es comprehender". En el estudio de Rosário et ál. (2006), esta categoría hace referencia al aprender como proceso reflexivo, que busca comprender el porqué de los acontecimientos. Es una búsqueda que se dirige tanto al material que se aprende como a distintas situaciones; hacia el mundo y hacia sí mismo.

En el estudiantado latinoamericano se agrega relacionar lo que se aprende con lo ya conocido; integrarlo en los esquemas mentales de cada uno o crear otros; reestructurar conocimientos anteriores -conductas que presuponen analizar-. Aprender, a mi entender, es poder comprender y relacionar los temas estudiados. Esto exige de cada uno mucha concentración (UNCuyo-TF-C8). Es la segunda conceptualización más mencionada por el estudiantado de las cuatro universidades ( $20 \%$ del total), en tres con mayor énfasis en el tramo inicial, mientras que en la UFRJ destacan en el tramo final.

Según universidad, su presencia es distintiva de la UdC y de la UNCuyo; en cuanto al recuento por tramo, prevalece en el inicial, aunque se encuentra también en el final.

6. "Aprender es ver las cosas de forma diferente". En Rosário et ál. (2006) esta categoría refiere al cambio en la forma de entender la realidad e incluye tanto la sustitución de una idea por otra como un cambio en la forma de pensar, con sentido amplio.

En el alumnado latinoamericano esta categoría se expresa como recrear y resignificar ideas; reestructurar pensamientos. Aprender para mí es significar y resignificar algo, es la búsqueda de certidumbres trabajando con incertidumbres y un proceso humanizador civilizatorio (UFRJ-TI-C22).

Desde la mirada de conjunto, solo representa el $6 \%$ del total y se reitera en la URFJ y la UNCuyo, como tendencia clara en el tramo final.

7. "Aprender es cambiar como persona". En Rosário et ál. (2006), el aprendizaje se vincula con un cambio personal y un desarrollo individual y social, lo que supone adquirir mayor madurez. 
http://doi.org/10.15359/ree.24-2.10

ROR: https://ror.org/01t466c14 Universidad Nacional, Costa Rica

http://www.una.ac.cr/educare

educare@una.cr

En el estudiantado latinoamericano esta categoría se expresa en términos semejantes, como un proceso interno, mediante el cual la persona desarrolla sus capacidades, se apropia de un nuevo actuar y pensar para enfrentar la realidad; una forma de superación y perfeccionamiento personal; el logro de una visión cada vez más acabada de sí. Proceso que puede desarrollarse tanto de manera autónoma, como así también bajo el acompañamiento de otra persona, donde el sujeto adquiere y desarrolla herramientas para su emancipación (UNCuyoTF-C25). Aprender es poder aplicar todo el bagaje de conocimientos que uno aprende a lo largo del tiempo a fin de desarrollar sus capacidades en pos de un desarrollo integral como persona (UNCuyoTF-C14). Aprender para mí significa construir un conocimiento basado y desarrollado por medio de la práctica y de la experiencia, este nos permite desarrollarnos como mejores individuos (UdCTF-C94). Esta categoría (que nuevamente representa el $6 \%$ del total) se observa principalmente en la UNCuyo, en ambos tramos, y es poco frecuente en los restantes contextos.

8. "Aprender es realizarse". En estudiantado portugués remite a las expectativas de quien aprende de lograr algo en el futuro.

En nuestro corpus se expresa como realización de sueños. Poner en práctica lo que es enseñado en teoría, tener acceso a las informaciones que están en nuestra vida, poder realizar sueños que están presentes en nuestros objetivos (UEP-TI-C2) y es infrecuente en el conjunto del estudiantado de las cuatro universidades. Solo aparece en la UNCuyo en dos producciones del tramo inicial y en la UEP en dos del inicial y una del final.

9. "Aprender es un proceso no limitado por el tiempo o el espacio". En cuanto surge en "una variedad de contextos cotidianos y escolares". Asimismo, se entiende "como un proceso continuo y gradual que se inicia en el nacimiento y termina en la muerte $y$, también, como un proceso infinito" ya que nadie "consigue acceder a la totalidad del conocimiento".

La concepción del aprendizaje como un proceso que abarca toda la vida es el modo en que esta categoría se hace presente en el estudiantado latinoamericano. Desde la perspectiva temporal, es un camino prolongado, extenso, inacabable. Es el conocimiento que se adquiere a través del tiempo (UdC-TF-C12). Para mí aprender es un camino bastante prolongado, extenso y que nunca termina. Aprender es integrar todo lo que yo tengo u obtengo como conocimiento, preguntándome sobre ello, pensándolo, interpretándolo, con el fin de ir más allá de lo que tengo, más allá de todo, interpretando ese conocimiento (UNCuyo-TI-C7).

Desde la perspectiva espacial, no solo se aprende en el aula sino en diversos ámbitos. Proceso de formar y construir conocimientos, experiencias en contextos formales y no formales de educación (UNCuyo-TF-C1).

Se menciona en las cuatro universidades ( $7 \%$ del total) con diferente énfasis. El estudiantado argentino es quien más empleó esta conceptualización, aunque su presencia es considerable en la UEP en el tramo final y mínima en la UdC (en proporción con el total de esta universidad) en ambos tramos. 
10. "Aprender es un proceso individualizado". En la muestra de estudiantado portugués, la idea central de esta categoría es que el sujeto tiene una forma propia de llevar a la práctica lo que aprende, situación en la que influyen factores internos del alumnado.

De modo equivalente, en estudiantado latinoamericano, aprender es un proceso que realiza cada persona e implica que lo aprendido se hace propio. Es ir modificando día a día conocimientos previos e ir construyendo nuevos saberes, nunca se deja de aprender, la manera en que aprendemos es individual (UdC-TI-C23). Esta categoría (que representa un $5 \%$ del total) se reitera en la UNCuyo en ambos tramos, mientras que en las otras universidades su frecuencia es mínima.

11. "Aprender es un proceso experiencial". En estudiantado portugués se aprende en numerosas situaciones de la vida cotidiana. El alumno o alumna construye activamente su aprendizaje sobre la base de la experiencia en diferentes circunstancias, de modo tal que el aprender adquiere un perfil holístico.

En nuestro estudio, no solo se aprenden experiencias sino que el acto mismo de aprender es una experiencia. Aprender para mí encierra una serie de preguntas como interactuar (el sujeto y el medio, el sujeto con otros) y dialogar, a fin de transformar experiencias y con eso enriquecer el capital cultural, social, económico, etc. (UFRJ-TF-C38). Esta categoría es infrecuente ( $2 \%$ del total): no aparece en la UNCuyo y manifiesta una ocurrencia en la UEP y dos en la UdC. Son estudiantes del tramo final de la UFRJ quienes la tienen en cuenta.

12. "Aprender es un proceso interactivo". El individuo aprende en interacción con otras personas y a través de ellas, en un proceso de intercomunicación del saber adquirido.

En el corpus latinoamericano también hallamos referencias al aprender como un proceso que realiza la persona con la compañía y la guía de un sujeto otro; acaece en la participación, en el diálogo con los otros seres. Aprender es para la vida y durante toda nuestra vida, aprendemos cuando enseñamos, aprendemos juntos. Nadie aprende solo, aprendemos con el mundo (UFRJTF-C2), Aprender es poder tomar el conocimiento dado y resignificarlo para la vida, generando participación, diálogo con el otro para que podamos de manera crítica reflexionar sobre aquello que aprendemos (UNCuyo-TF-C2).

Esta concepción se encuentra en las producciones de las cuatro universidades (4 \% del total), con énfasis en la UEP en ambos tramos, seguida proporcionalmente por la UFRJ en el tramo final. En los otros contextos educativos su presencia es mínima, a veces en el tramo inicial y otras veces en el final.

13. "Aprender es un proceso de enseñanza". El aprendizaje se entiende en relación con un contexto de enseñanza formal, en el cual se desempeñan especialistas en educación. 
http://doi.org/10.15359/ree.24-2.10

ROR: https://ror.org/01t466c14 Universidad Nacional, Costa Rica

http://www.una.ac.cr/educare

educare@una.cr

En nuestra investigación se expresa como encuentro e interacción estudiante-docente. Es un proceso que realiza la persona 'educando' con la compañía y la guía de un educador (UNCuyo$\mathrm{TI}-\mathrm{C5}$ ). Esta categoría, al igual que la anterior, se encuentra en los cuatro contextos académicos en proporción baja ( $5 \%$ del total). Adquiere una presencia más relevante en la UdC en el tramo final, pero también se observa en la UFRJ y UdC.

14. "Aprender es positivo". Estudiantes de la muestra portuguesa piensan el aprendizaje como algo que es importante y que gratifica.

En nuestro estudio, aprender (...) Ileva todo un proceso de enseñanza aprendizaje que es muy importante (UdC-TF-C9). Es conocer el sentido de algo que ayuda a mejorar la vida de la gente (UEPTI-C15). Esta categoría solo se reconoce en las dos producciones consignadas (pp. 199-200).

\section{Nuevas categorías en estudiantado latinoamericano: Solapamientos y situaciones particulares emergentes de la comparación}

Durante la aplicación del sistema de categorías de Rosário et ál. (2006) al corpus, observamos algunas concepciones que se solapan -sin quedar abarcadas en su totalidad- y otras propias, distintivas de nuestra población.

En un análisis que mantiene la lógica del precedente (cfr. Tabla 3), ponderamos el comportamiento de las segundas.

Tabla 3: Síntesis de resultados para categorías nuevas

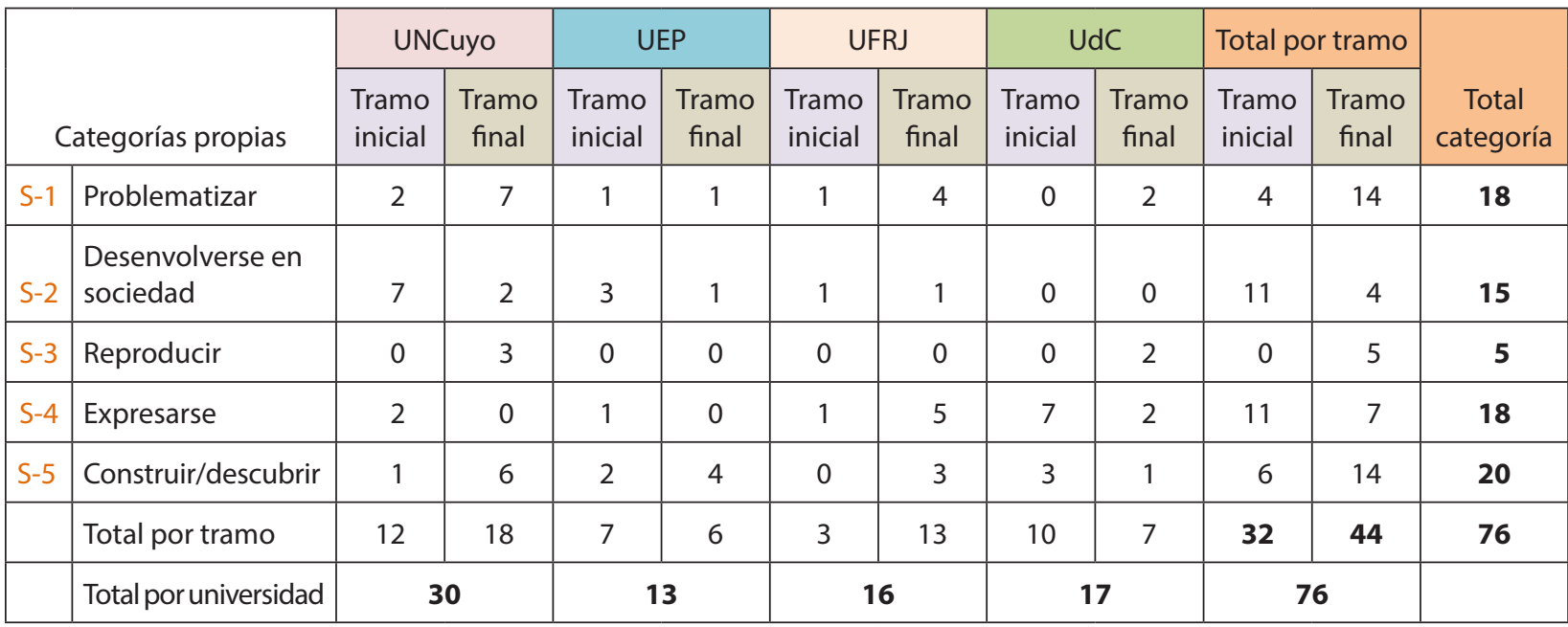

Nota: Elaboración propia. 
Una categoría de Rosário et ál. (2006) que resulta parcialmente apropiada para nuestro corpus es aprender es comprehender, debido a que no contempla aspectos tales como problematizar y cuestionar, los que se reiteran en las producciones del estudiantado latinoamericano, en particular en el tramo final en la UNCuyo y la UFRJ. Por ello podría agregarse una nueva categoría:

- Aprender es problematizar, la que incluiría expresiones tales como cuestionar, interpretar y reflexionar. Por ejemplo: Aprender es disponer del conocimiento para problematizarlo, cuestionarlo y reflexionarlo, tener la posibilidad de recrear, resignificar y expresar ideas (UNCuyo-TF-C3). Aprender es desarrollar la capacidad de argumentar y cuestionar críticamente algo que fue construido durante un proceso de enseñanza-aprendizaje (UFRJTF-C1). Esta categoría se muestra, principalmente, en el tramo final, en la UNCuyo y la UFRJ y, en menor proporción, en el inicial y en los restantes contextos académicos.

Otras categorías que sería necesario incorporar, a fin de abarcar en forma exhaustiva las concepciones de aprendizaje de la muestra estudiada, son:

- Aprender es desenvolverse en la sociedad. Observamos que algunos sujetos participantes emplearon expresiones que aluden a conocer lo que sucede en el entorno, explorarlo, transformarlo, a fin de adaptarse y sociabilizarse. Esta observación se apoya en producciones de diferentes unidades de análisis y en los dos tramos, entre ellas: Es crecery buscar un desarrollo para vivir en sociedad y tener más oportunidades (UEP-TI-C8). Proceso por el cual nos apropiamos de una serie de saberes (actitudinales, procedimentales, conceptuales) que nos servirán en el desenvolvimiento de cada uno de los sujetos (en la sociedad en general, en diferentes campos, en diferentes contextos) (UNCuyo-TF-C21). Es más frecuente en la UNCuyo, tramo inicial y en menor proporción, en la UEP y la UFRJ. Solo está ausente en la UdC.

- Aprender es reproducir lo aprendido. Aunque Rosário et ál. (2006) incluyen la concepción identificada como aprenderes memorizar, aclaran que se refiere a una forma de almacenar la información que no prevé su reproducción. Por el contrario, como en Säljö (1979), fijar y reproducir se menciona en las producciones de nuestros estudiantes: [Aprender] es un proceso a través del cual adquieres conocimientos, ya sea teóricos o prácticos, los cuales puedes reproducir o aplicar posteriormente (UdC-TF-C17). Es incorporar información nueva y poder reproducirla luego de haber comprendido el material (UNCuyo-TF-C35). Esta categoría se muestra explícitamente en cinco producciones de estudiantes que se encuentran en el tramo final en la UNCuyo y en la UdC.

- Aprender es poner en palabras propias lo aprendido. En nuestra población, un grupo de estudiantes identifica el aprendizaje con la capacidad de verbalizar; ello sugiere la necesidad de evocar y comunicar el conocimiento como indicadores de aprendizaje efectivo. Si bien en la categoría aprender es un proceso interactivo se halla implícita 
http://doi.org/10.15359/ree.24-2.10

ROR: https://ror.org/01t466c14 Universidad Nacional, Costa Rica

http://www.una.ac.cr/educare

educare@una.cr

la transmisión, en ese caso, el énfasis se pone en el encuentro; en cambio, en la que proponemos, se centra en la producción personal. Aprender es cuando aquella exposición de tema visto queda grabada y se puede expresar el contenido sin dificultad ... asociándolo con demás temas (UdC-TF-C35). Aprender es asimilar algún contenido y poder explicar el contenido con otras palabras (UFRJ-TI-C23).

Esta categoría se reitera en estudiantes de la UdC, tramo inicial, y de la UFRJ, tramo final, mientras que en los casos restantes su proporción es mínima o está ausente.

- Es construir/descubrir algo nuevo. En el estudiantado latinoamericano es reiterada la referencia al aprender como la relación entre lo nuevo y lo conocido, una integración superadora de las ideas previas en las nuevas experiencias, un abrirse a realidades inéditas que permitan seguir aprendiendo. Aprender es descubrir algo que no se sabía antes, aprender para mí está ligado a diversión e interés personal en el asunto. Aprender por tanto es un recorrido en busca de lo desconocido (UFRJ-TF-C19). Aprender para mí significa construir un conocimiento basado y desarrollado por medio de la práctica y de la experiencia, este nos permite desarrollarnos como mejores individuos (UdC-TF-C39). Está presente en las cuatro universidades y, a excepción de la UFRJ, en ambos tramos, con mayor énfasis en el tramo final.

En cuanto al solapamiento entre categorías, en función de nuestro análisis, no encontramos diferencias claras entre aprender es cambiar como persona y aprender es realizarse. Ambas destacan la transformación y el desarrollo personal de cara al futuro, por lo que consideramos apropiado unificarlas en la categoría Aprender es cambiar como persona; por ejemplo: Aprender para mí es adquirir las habilidades competentes que me permiten perfeccionarme como persona en todas mis dimensiones, es decir de modo integral; conformando una visión valorativa propia, auténtica y realista (UNCuyo-TI-C15).

Tampoco resulta precisa la diferencia entre aprender es algo envolvente y diversificado y aprender es un proceso experiencial. Las dos están remitiendo al aprender como experiencia de la persona total, con sentido holístico, lo que sugiere la conveniencia de agruparlas en una sola categoría; por ejemplo: Es un proceso de construcción del conocimiento que se da a lo largo de toda la vida, en conjunto con otros sujetos y en diferentes ámbitos de vida, donde intervienen factores sociales, culturales, económicos, entre otros (UNCuyo-TF-C20).

Por último, en el conjunto de las producciones se reconocieron términos que adquieren mayor énfasis en una universidad en particular respecto de los restantes.

- Individualizado. Caracteriza a la UNCuyo y se vincula con el empleo más frecuente que en los otros contextos académicos de términos específicos del campo del saber: asimilación, acomodación, reestructuración, modificación conocimientos previos, metacognición, entre otros. 
- Nuevo. Se reitera de un modo llamativo en la UdC tramo inicial, en 55 participantes se encuentra 12 veces y en tramo final, en 40 casos se encuentra en 13 producciones.

- Adquirir/adquisición y aplicar/aplicación. También en la UdC adquiere un énfasis que lo posiciona como idiosincrático de este contexto académico.

- Interacción. En UFRJ y UEP -estudiantao brasileño-, se encuentran más referencias que en los otros dos contextos académicos a aspectos sociales que hacen al aprender.

Otra situación particular remite al procedimiento, propio de la investigación cualitativa, de recuperar todas las categorías. Dado el tamaño de la muestra, un listado exhaustivo resulta inoportuno; por tal razón, con sentido ejemplificativo incluimos solo algunas: aprender es entendido como derecho, proceso natural de todo ser humano, capacidad innata, creación de nuevas estructuras cognitivas, aproximación a la verdad y a nosotros mismos, entre otras.

\section{Convergencias y divergencias en las concepciones de aprender entre estudiantado latinoamericano y portugués}

En ambas investigaciones, las categorías que más se reiteran son incrementar el conocimiento y comprehender y son poco mencionadas, aprender es positivo y aprender es realizarse (obtener algo proyectado hacia el futuro).

Luego del análisis descriptivo por categoría, Rosário et ál. (2006) incluyen una lectura más global de sus resultados, la que ayuda a plantear las convergencias. Desde esta perspectiva se reconocen tres aspectos (Grácio, 2003, en Rosário et ál., 2006): qué es aprender, cómo se lleva a cabo y qué valor tiene.

El primero se compone, a su vez, por tres focos:

"Extensión y diversidad" (aprender es algo abarcable y diversificado en cuanto a contenidos, procesos y fuentes de aprendizaje); "Instrumental" (aprender es incrementar los conocimientos, memorizar y aplicar); "Transformativo" (aprender es comprender, ver las cosas de forma distinta, cambiar como persona y realizarse). (p. 202).

En la muestra latinoamericana, en función de los datos consignados en la Tabla 2, el esquema precedente remite al siguiente análisis por tramo:

- Envolvente: equivalencia entre tramos (T inicial/T final $=13$ )

- Instrumental: mayor énfasis en el tramo inicial ( $\mathrm{T}$ inicial $=98$, T final $=83$ )

- Transformativo: la frecuencia es superior en el tramo inicial, pero el porcentaje resulta cercano al del tramo final ( $T$ inicial $=85, T$ final $=70$ ). 
Como en la muestra del estudiantado portugués, también en nuestros resultados se halla una tendencia a la disminución de las concepciones referidas al foco instrumental en el tramo final. Dado que la proporción de transformativas no se modifica sustancialmente, se podría inferir una relativa complejidad creciente durante la trayectoria formativa.

Cómo se lleva a cabo, incluye las referencias al proceso y se compone de cuatro focos, cuya denominación anticipa el contenido: Espacio-temporal, individualización, experiencial y mediación social y enseñanza.

En el estudiantado latinoamericano, la situación es:

- Espacio-temporal: casi equivalente entre tramos ( $T$ inicial $=16, T$ final $=17$ )

- Individualización: predomina en el tramo inicial ( $T$ inicial =14, T final = 8)

- Experiencial: escasa y uniforme entre tramos ( $T$ inicial $=4, T$ final $=5)$

- Mediación social y enseñanza: ambas categorías predominan en el tramo final (T inicial $=6, \mathrm{~T}$ final $=12 ; \mathrm{T}$ inicial $=7$, T final $=15$; porcentaje combinado $=5 \%$ y $12 \%$, respectivamente).

La mirada de conjunto de los resultados parciales en las categorías representativas de este aspecto muestra que son heterogéneos, con leve inclinación en el tramo final a incluir en la concepción referencias al cómo se realiza el proceso de aprender.

Valor que se atribuye a aprender, remite a la importancia del aprendizaje, su valor positivo ( T inicial = 1 y T final = 1). En este aspecto, en consonancia con Rosário et ál. (2006), las referencias explícitas a esta categoría son mínimas.

\section{Conclusiones}

El presente artículo informa los resultados de una investigación que se realizó en una muestra conformada por 264 estudiantes que cursan el $2^{\circ}$ y el último año (tramo inicial/final) en Ciencias de la Educación/Pedagogía en la UNCuyo (Argentina), la UFRJ y la UEP (Brasil) y la UdC (México).

Retomando el objetivo del estudio -ponderar los alcances y límites de aplicar el sistema categorial de Rosário et ál. (2006) a las concepciones de aprender extraídas de diferentes universidades latinoamericanas-, se puntualizaron las concepciones presentes en los dos casos -completa o parcialmente- y las que se encuentran en uno, pero no en el otro, con ello quedan a la vista nuevas categorías en función de los resultados obtenidos en las universidades mencionadas. 
Por un lado, el referido sistema (en catorce categorías) resultó funcional para analizar lo que entiende por aprender el estudiantado universitario en diferentes instituciones y países. En este sentido, las concepciones identificadas en alumnado portugués están presentes, en mayor o menor proporción, en el estudiantado argentino, brasileño y mexicano que participó en nuestra investigación, lo que sugiere que las concepciones del aprender se definen en sentidos que atraviesan diferentes contextos académicos. Ello conduciría a alejarnos de la idea de que las conceptualizaciones de aprendizaje son idiosincráticas según contexto.

No obstante, por otro lado, las discrepancias halladas en las producciones del alumnado latinoamericano entre sí y con el estudiantado portugués hacen notar el riesgo de considerar sin más que las concepciones son transversales, generalizables a diferentes contextos académicos. Al respecto, el sistema categorial de Rosário et ál. (2006) no resulta suficiente para abarcar todas las concepciones de aprendizaje que identificamos. Aprender es problematizar, desenvolverse en la sociedad, expresar lo aprendido, construir/descubrir algo nuevo, que son reiteradas en la muestra latinoamericana no aparecen en Rosário et ál. (2006).

En consecuencia, nos inclinamos por una postura que reconoce semejanzas y solapamientos, a la vez que subraya particularidades en función del contexto educativo. Sin embargo, en ambas muestras las concepciones de aprendizaje (como en Martínez-Fernández, 2007; Pozo y Scheuer, 1999; Pozo et ál., 2009) incluyen algunas reproductivas -aprender es saber más, adquirir conocimientos-, otras interpretativas -aprender es realizar actividades para procesar la información dada-y otras constructivas -aprender es transformar lo dado y cambiar como persona-.

Respecto del tramo, si bien el macroanálisis desde los tres aspectos propuestos por las personas autoras mostró tendencias, su alcance resulta poco claro, lo que lleva a pensar que a la base de lo que el alumnado universitario entiende por aprender, más que el tramo de la trayectoria educativa en que se encuentra, gravitan factores personales -por ejemplo, estudiantado más o menos analítico/reflexivo; más/menos inquieto intelectualmente, ingenioso, inclinado al pensamiento divergente- y factores académicos. Al respecto, conocer y tener en cuenta las concepciones de aprendizaje constituye el primer paso para la construcción de aprendizajes significativos, la prevención de fracasos inexplicados y frustraciones personales que pueden llevar al abandono de la carrera.

Entre los factores académicos, se pueden enumerar: antecedentes de interacciones con el personal docente, en particular, respecto del clima que se vive en el aula, el que puede (o no) generar un respeto incondicional a puntos de vista divergentes; la implementación de propuestas didácticas que motiven el pensamiento autónomo; la experiencia del tipo de evaluación -lo que remite a aquello que el profesorado entiende por aprender-; la confianza en la capacidad de quien aprende de pensar alternativas que transformen en algún sentido 
el contenido dado y la expectativa de que, en el curso de la carrera, se produzca un avance en dirección a miradas críticas y reflexivas. De allí que generar instancias en clase para que el estudiante haga conscientes y describa las conductas que emplea para aprender en su relación con la concepción de aprendizaje que la sustenta posibilita procesos metacognitivos y de autorregulación, que van a incidir en el desempeño académico.

Por otra parte, tanto la muestra portuguesa como la latinoamericana está conformada por estudiantado universitario que se prepara para ser docente. Por esta razón, es preciso indagar y debatir en el encuentro pedagógico las implicaciones de la concepción de aprendizaje de cada estudiante por el sentido que adquiere para cada profesional del campo de la educación reconocerlas en la propia conducta, a fin de evitar repetir modelos sin reflexión.

Además, las concepciones de aprendizaje aportan explicaciones sobre los modos de llevar a la práctica el aprendizaje y, en vistas a que se preparan para ser educadores y educadoras, representan un contenido específico que es necesario trabajar en el plano conceptual y procedimental. Es necesario que el profesorado haga referencia a las formas alternativas de aprender los diferentes contenidos que presenta y que señale las diferencias respecto de la posibilidad de aplicar y transferir lo que se adquirió de un modo repetitivo, respecto de lo que se aprendió según una mirada crítica y de innovación.

Por último, en el estudio de las concepciones del aprender, la posibilidad de generalizar resultados es necesariamente prudencial a fin de compatibilizar categorías comprehensivas con aspectos diferenciales que permitan representar lo distintivo del corpus en análisis y respetar el entramado peculiar de la respuesta de cada estudiante.

\section{Algunas implicaciones}

Como ha quedado de manifiesto, a la hora de pensar el acto educativo, es relevante tener en cuenta las concepciones de aprendizaje de quienes intervienen en este, en tanto factor explicativo de su dinámica.

Esto se pudo apreciar el 6 de octubre de 2017, en un encuentro científico en la Universidad Nacional de Córdoba, Argentina. El equipo que lleva adelante la investigación en la UNCuyo presentó una ponencia, cuyo contenido remite a la temática aquí desarrollada. Los planteos expuestos fueron adoptados como puntos de referencia para explicar resultados en otras presentaciones en las que se informaba sobre experiencias pedagógicas en contextos de privación de la libertad y en educación hospitalaria.

El debate que siguió a las ponencias ubicó en primer plano la incidencia de las concepciones en el obrar del estudiantado y, como complemento, la influencia de la propuesta pedagógico-didáctica en la formación de concepciones en el alumnado, de las que, a la vez,

\footnotetext{
20 Ida Lucía Morchio, Solange Astudillo e Hilda Difabio-de Anglat
}

Los artículos de la Revista Electrónica Educare del Centro de Investigación y Docencia en Educación de la Universidad Nacional, Costa Rica, se comparten bajo términos de la Licencia Creative Commons: Reconocimiento, № Comercial, Sin Obra Derivada 3.0 Costa Rica. Las autorizaciones adicionales a las aquí delimitadas se pueden obtener en el correo: educare@una.cr 
derivan diversos modos de organización de la conducta. Esto es, las concepciones adquieren un sentido bi-direccional. Por un lado, se inician en el estudiantado y condicionan su actuar frente a la propuesta didáctica docente. Por otro, esta propuesta didáctica, entendida como la enseñanza efectiva, incide en lo que el estudiantado entiende por aprender.

En dicha propuesta, la evaluación del aprendizaje se posiciona en un lugar destacado. Si quien enseña prioriza el retener y recordar información, es probable que, en la concepción del estudiantado, el aprender adquiera una lógica de reproducción. En cambio, si los objetivos del personal docente incluyen transferir lo aprendido a situaciones variadas y analizar el contenido dado con sentido crítico, si valora la creatividad y el pensamiento autónomo, la concepción del estudiantado se formalizará, siguiendo a González Cabanach (1997), hacia la comprensión significativa y la posibilidad de vincularla con los propios planes personales, como así también será diferente su anticipación de sentido respecto de los criterios con que será evaluado.

En suma, se genera un proceso dialéctico entre lo que el estudiantado entiende por aprender y la propuesta pedagógica-didáctica que implementa el profesorado; ambos aspectos se condicionan mutuamente. Sería interesante en futuras investigaciones ponderar en qué medida y de qué modo interactúan.

\section{Referencias}

Bruner, J. S. (2001). Desarrollo cognitivo y educación. Morata.

Cano, F. (2005). Consonance and dissonance in students' learning experience. Learning and Instruction, 15(3), 201-223. https://doi.org/10.1016/j.learninstruc.2005.04.003

Cuberos Pérez, M., Santamaría Santigosa, A., Prados Gallardo, M. del M. y Arias Sánchez, S. (2019). Concepciones del aprendizaje de estudiantes en proceso de formación como docentes. Profesorado. Revista de currículum y formación del profesorado, 23(3), 453-471. https://doi. org/10.30827/profesorado.v23i3.11238

González Cabanach, R. (1997). Concepciones y enfoques de aprendizaje. Revista de Psicodidáctica, 4, 5-39. http://www.redalyc.org/articulo.oa?id=17517797002

Martínez-Fernández, R. (2007). Concepción de aprendizaje y estrategias metacognitivas en estudiantes universitarios de Psicología. Anales de Psicología, 23(1), 7-16. http://revistas. um.es/analesps/article/view/23261

Marton, F. y Säljö, R. (1976). On qualitative differences in learning. I. - outcome and process. British Journal of Educational Psychology, 46(1), 4-11. https://doi.org/10.1111/j.2044-8279.1976. tb02980.x 
Marton, F., Dall'Alba, G. y Beaty, E. (1993). Conceptions of learning. International Journal of Educational Research, 19(3), 277-300.

Morchio, I. L. (Dir.). (2016-2018). Aproximación pluridimensional al aprender en la universidad. Hacia una gestión autorregulada del aprendizaje (Proyecto código 06/G746). http://www.uncuyo. edu.ar/ciencia tecnica y posgrado/catalogo investigacion/proyecto/buscar?unidad academica id=9\&convocatoria id=\&proyecto=\&integrante=morchio

Morchio, I. L. y Del Río, A. (2015). Concepciones de aprender en alumnos universitarios de cinco carreras. En I. L. Morchio (Coord.), Aprender a aprender como meta de la educación superior. Desde la comprensión de cómo aprende el alumno universitario a la promoción del aprendizaje autorregulado (pp. 81-120). Buenos Aires: Teseo. https://www.editorialteseo. com/archivos/13535/aprender-a-aprendercomo-meta-de-la-educacion-superior/

Morchio, I. L. y Difabio de Anglat, H. (2018). Inventario de concepciones y experiencias de aprender en la universidad. Estructura, contenido y propiedades psicométricas. Revista de Psicología, 14(27), 25-55. http://bibliotecadigital.uca.edu.ar/greenstone/cgi-bin/library. cgi? $\mathrm{a}=\mathrm{d} \& \mathrm{c}=$ Revistas\&d=inventario-concepciones-experiencias-aprender

Pozo, J. I. y Scheuer, N. (1999). Las concepciones sobre el aprendizaje como teorías implícitas. En J. I. Pozo y C. Monereo (Coords.), El aprendizaje estratégico. Enseñar a aprender desde el currículo (pp. 87-108). Santillana.

Pozo, J. I., Scheuer, N., Pérez, M., Mateos, M., Martín, E. y de la Cruz, M. (2009). Nuevas formas de pensar la enseñanza y el aprendizaje. Las concepciones de profesores y alumnos. Graó.

Rosário, P., Grácio, M. L., Núñez, J. C. y González-Pienda, J. (2006). Perspectiva fenomenográfica de las concepciones del aprendizaje. Revista Galego-Portuguesa de Psicoloxía e Educación, 13(11-12), 195-206. http://ruc.udc.es/dspace/bitstream/handle/2183/7024/RGP 13-10. pdf?sequence $=1$

Säljö, R. (1979). Learning about Learning. HigherEducation, 8(4), 443-451.https://doi.org/10.1007/ $\underline{B F 01680533}$ 